\title{
Molecular sexing of tigers, Panthera tigris
}

\author{
R. McEwing $\cdot$ K. Ouitavon $\cdot$ J. J. Rovie-Ryan • \\ Wulansari $\cdot$ F. T. Sitam $\cdot$ R. Ogden
}

Received: 27 June 2011/ Accepted: 6 September 2011

(C) Springer Science+Business Media B.V. 2011

\begin{abstract}
We report the development of a fast and reliable PCR-based method for sex identification of tiger DNA designed to be incorporated into fluorescent short tandem repeat (STR) profiling. A single primer pair, consisting of a fluorescently-labelled forward primer and an unlabelled reverse primer, is used to co-amplify homologous fragments of a zinc finger (ZF) protein intron which exhibits size polymorphism between the $\mathrm{X}$ and $\mathrm{Y}$ chromosomes. The ZFX and ZFY amplicons differ in size by $12 \mathrm{bp}$ and can thus be differentiated by capillary electrophoresis.
\end{abstract}

Keywords Molecular sex identification - Tiger ·

Zinc finger protein $\cdot$ Forensics $\cdot$ Felids

\section{Introduction}

Tiger parts are highly sought after, primarily as an ingredient in traditional Asian medicine and as ornamental skins. The

R. McEwing $(\bowtie) \cdot R$. Ogden

Royal Zoological Society of Scotland, 134 Corstorphine Road, Edinburgh EH12 6TS, Scotland, UK

e-mail: ross.mcewing@ tracenetwork.org

K. Ouitavon

DNP Wildlife Forensic Science Unit, Wildlife Conservation

Office, Department of National Parks, Wildlife and Plant

Conservation, 61 Phaholyothin Rd., Ladyao, Chatuchak,

Bangkok 10900, Thailand

J. J. Rovie-Ryan · F. T. Sitam

Wildlife Genetic Resource Bank (WGRB) Laboratory,

Department of Wildlife and National Parks,

Km. 10, Jalan Cheras, 56100 Kuala Lumpur, Malaysia

Wulansari

Eijkman Institute for Molecular Biology, Diponegoro 69,

Jakarta 10430, Indonesia demand for tiger parts along with habitat destruction was the principle cause for the decline in tiger numbers during the twentieth century, and despite international legislation and domestic bans on tiger part trade, poaching and illegal activity continue to threaten the world's tigers today (Linkie et al. 2003). Six extant subspecies of tiger are currently recognized, Panthera tigris tigris, Panthera tigris corbetti I, Panthera tigris corbetti II (jacksoni/malayensis), Panthera tigris altaica, Panthera tigris sumatrae and Panthera tigris amoyensis, and all are listed as 'endangered' or 'critically endangered' on the IUCN Red List of Threatened Species (IUCN 2010). Population monitoring of these species, including sex ratio data, is therefore vital to provide information for their conservation and management. Although the sex of adult tigers can be determined by an experienced observer, field observations may be hampered by distance, position relative to the animal or by vegetation. Furthermore, juvenile tigers are difficult to sex, and illegally traded parts and derivatives of tigers have no sexual characters.

Here, we report the development of a non-invasive molecular sexing technique for tiger DNA. In this study, we exploit a size difference ( $\sim 12 \mathrm{bp}$ ) between ZFX and ZFY homologs, elucidated by capillary electrophoresis, to determine the sex of tiger samples. The current research forms part of a larger project to develop genetic markers for linking tiger parts to their individual source animal, in order to help investigate illegal poaching and trade of tigers, and their parts.

\section{Methods}

Samples

Samples of blood or hair were collected from zoo tigers of known sex $($ male $=15$, female $=17$ ) from four of the six 